\title{
Introduction of a standardized multimodality image protocol for navigation-guided surgery of suspected low-grade gliomas
}

\author{
Aygül Mert, MD, ${ }^{1}$ Barbara Kiesel, MD, ${ }^{1}$ Adelheid Wöhrer, MD, PhD, ${ }^{2}$ \\ Mauricio Martínez-Moreno, MD,, Georgi Minchev, MD, ${ }^{1}$ Julia Furtner, MD, ${ }^{3}$ Engelbert Knosp, MD,1 \\ Stefan Wolfsberger, MD, ${ }^{1}$ and Georg Widhalm, MD, PhD'
}

\begin{abstract}
'Department of Neurosurgery, ${ }^{2}$ Institute of Neurology, and ${ }^{3}$ Department of Biomedical Imaging and Image-guided Therapy, Medical University Vienna, Austria
\end{abstract}

OBJECT Surgery of suspected low-grade gliomas (LGGs) poses a special challenge for neurosurgeons due to their diffusely infiltrative growth and histopathological heterogeneity. Consequently, neuronavigation with multimodality imaging data, such as structural and metabolic data, fiber tracking, and 3D brain visualization, has been proposed to optimize surgery. However, currently no standardized protocol has been established for multimodality imaging data in modern glioma surgery. The aim of this study was therefore to define a specific protocol for multimodality imaging and navigation for suspected LGG.

METHODS Fifty-one patients who underwent surgery for a diffusely infiltrating glioma with nonsignificant contrast enhancement on MRI and available multimodality imaging data were included. In the first 40 patients with glioma, the authors retrospectively reviewed the imaging data, including structural MRI (contrast-enhanced T1-weighted, T2-weighted, and FLAIR sequences), metabolic images derived from PET, or MR spectroscopy chemical shift imaging, fiber tracking, and 3D brain surface/vessel visualization, to define standardized image settings and specific indications for each imaging modality. The feasibility and surgical relevance of this new protocol was subsequently prospectively investigated during surgery with the assistance of an advanced electromagnetic navigation system in the remaining 11 patients. Furthermore, specific surgical outcome parameters, including the extent of resection, histological analysis of the metabolic hotspot, presence of a new postoperative neurological deficit, and intraoperative accuracy of 3D brain visualization models, were assessed in each of these patients.

RESULTS After reviewing these first 40 cases of glioma, the authors defined a specific protocol with standardized image settings and specific indications that allows for optimal and simultaneous visualization of structural and metabolic data, fiber tracking, and 3D brain visualization. This new protocol was feasible and was estimated to be surgically relevant during navigation-guided surgery in all 11 patients. According to the authors' predefined surgical outcome parameters, they observed a complete resection in all resectable gliomas $(n=5)$ by using contour visualization with T2-weighted or FLAIR images. Additionally, tumor tissue derived from the metabolic hotspot showed the presence of malignant tissue in all WHO Grade III or IV gliomas ( $n=5)$. Moreover, no permanent postoperative neurological deficits occurred in any of these patients, and fiber tracking and/or intraoperative monitoring were applied during surgery in the vast majority of cases $(n=10)$. Furthermore, the authors found a significant intraoperative topographical correlation of 3D brain surface and vessel models with gyral anatomy and superficial vessels. Finally, real-time navigation with multimodality imaging data using the advanced electromagnetic navigation system was found to be useful for precise guidance to surgical targets, such as the tumor margin or the metabolic hotspot.

CONCLUSIONS In this study, the authors defined a specific protocol for multimodality imaging data in suspected LGGs, and they propose the application of this new protocol for advanced navigation-guided procedures optimally in conjunction with continuous electromagnetic instrument tracking to optimize glioma surgery.

http://thejns.org/doi/abs/10.3171/2014.10.FOCUS14597

KEY WORDS suspected low-grade gliomas; neuronavigation; standardized protocol; multimodality image data

ABBREVIATIONS CSI = chemical shift imaging; DTI = diffusion tensor imaging; fMRI = functional MRI; HGG = high-grade glioma; LGG = low-grade glioma; MRS = MR spectroscopy; RANO = Response Assessment in Neuro-Oncology; ROI = region of interest; T/N = tumor-to-normal brain ratio; TSE = turbo spin echo.

SUBMITTED September 1, 2014. ACCEPTED October 7, 2014. INCLUDE WHEN CITING DOI: 10.3171/2014.10.FOCUS14597.

DISCLOSURE Stefan Wolfsberger is currently an educational consultant and a technological advisory board member of Medtronic. All other authors report no conflict of interest concerning the materials or methods used in this study or the findings specified in this paper. 
$\mathrm{O}$ F all primary CNS neoplasms, diffusely infiltrating gliomas represent the most frequently occurring tumor entity. ${ }^{2}$ If glioma is suspected, the imaging method of choice is MRI ${ }^{4}$ After administration of contrast medium, low-grade gliomas (LGGs; WHO Grade II) usually show nonsignificant contrast enhancement on MRI, whereas high-grade gliomas (HGGs; WHO Grades III and IV) generally demonstrate significant contrast enhancement. ${ }^{13,21,35}$ Whereas HGG are routinely treated with radio- and/or chemotherapy after neurosurgical resection or biopsy,,$^{21,32}$ the primary goal in the initial treatment of LGG is maximum safe resection in the majority of patients. ${ }^{24,28,29}$ Therefore, optimizing glioma surgery is indispensable in LGGs as these tumors are generally treated by surgery alone.

However, surgical treatment of suspected LGGs poses a special challenge for the neurosurgeon for the following reasons: 1) Incomplete glioma resection. Due to their diffusely infiltrative growth pattern, intraoperative identification of the exact tumor border in an LGG is frequently not possible with certainty. Thus, incomplete glioma resection is not uncommon and has been reported in up to $88 \%$ of cases. ${ }^{3,24,25,28}$ However, there exists mounting evidence that extensive resection of LGGs is associated with prolonged overall survival., ${ }^{924,28}$ Recently, the Response Assessment in Neuro-Oncology (RANO) Working Group defined the goal of surgery in LGGs as total removal of the entire abnormality on preoperative FLAIR/T2-weighted sequences. ${ }^{35,36}$ 2) Histopathological undergrading. Progression of tumors that are initially LGG generally occurs within focal intratumoral areas of malignant transformation referred to as anaplastic foci. ${ }^{11,19,26}$ This tissue heterogeneity with benign and malignant areas within the same glioma could lead to histological undergrading if tissue samples are taken from random tumor areas. ${ }^{19}$ Therefore, the surgical goal is to perform precise tissue sampling from a potential anaplastic focus to avoid histopathological undergrading and subsequent treatment failure. 3) Eloquent tumor localization. LGGs are characterized by their infiltrative growth pattern and are commonly located adjacent to eloquent brain areas. ${ }^{7.9}$ Maximum tumor resection therefore carries the risk of new postoperative neurological deficits. Thus, the goal of surgery is to identify and preserve such functional brain areas during glioma resection. 4) Insufficient comprehension of cortical surface anatomy and vasculature. Topographic localization of the tumor and its relation to the brain surface and superficial vasculature on standard 2D MR images requires a high level of experience to define precisely. ${ }^{14}$ Inaccurate localization of these structures might result in increased perioperative morbidity. Therefore, the neurosurgeon needs to precisely localize the tumor and its relation to cortical surface anatomy and vasculature in the preoperative planning phase as well as during the operative approach.

To achieve the aforementioned surgical goals that are highly relevant for patient prognosis and outcome, promising imaging modalities that are integrated into neuronavigation systems have been clinically validated in the last few years and have been proposed to optimize glioma surgery. First of all, navigation with structural MRI represents a powerful tool for improved visualization of the tu- mor border in relation to adjacent anatomy to increase the rate of complete tumor resection. ${ }^{12,40}$ Furthermore, navigation with metabolic imaging using PET and/or MR spectroscopy (MRS) chemical shift imaging (CSI) is a clinically reliable method for intraoperative identification of anaplastic foci to avoid histopathological undergrading of gliomas. ${ }^{20,30,34,38}$ Moreover, navigation with diffusion tensor imaging (DTI) is capable of identifying functionally relevant white matter tracts adjacent to the glioma with the aim of minimizing the risk of a new postoperative neurological deficit..$^{1,5,17}$ Finally, our group demonstrated recently that the application of 3D brain surface visualization within neuronavigation is able to identify the exact anatomical glioma and vasculature localization in the preoperative planning phase and during glioma surgery. ${ }^{14}$ Throughout the last decade, advanced neuronavigation systems have been developed that are capable of integrating and visualizing data from multiple imaging modalities in a single picture. ${ }^{18}$ However, no consensus exists regarding a specific imaging protocol for neuronavigation that fulfills the specific requirements of modern glioma surgery. Thus, such a protocol for imaging and navigation setup for surgery of suspected LGGs is needed.

The aim of the present study was therefore to define a multimodality imaging protocol for diffusely infiltrating gliomas with nonsignificant contrast enhancement on MRI with standardized image settings and specific indications for each modality. Subsequently, this new multimodality imaging protocol was prospectively clinically validated during advanced navigation-guided surgery for suspected LGGs.

\section{Methods}

Fifty-one patients with a diffusely infiltrating glioma with nonsignificant contrast enhancement on MRI have been surgically treated since 2008 with the aid of multimodality imaging navigation at the Department of Neurosurgery, Medical University Vienna. With the introduction of a new navigation system in our department in 2011, we integrated the multiple imaging data of the first 40 patients into our planning station and retrospectively reviewed these data to define standardized imaging settings and specific indications for each imaging modality. These data served as the basis for a multimodality imaging protocol that was then prospectively applied and clinically validated in the remaining 11 patients. The patient characteristics are provided in Tables 1 and 2 . This study was approved by the local ethics committee, and patients gave informed consent.

\section{Preoperative Imaging}

The following imaging modalities were performed within 4 weeks prior to surgery in each patient.

\section{Structural Imaging}

All preoperative MRI was conducted using a 3-T clinical scanner (Tim Trio, Siemens). The following structural imaging sequences were performed: T1-weighted images after contrast medium administration, T2-weighted and FLAIR sequences. In the current study, only gliomas with nonsignificant contrast enhancement (none, patchy, 
TABLE 1. Patient characteristics

\begin{tabular}{|c|c|}
\hline Variable & Value $^{*}$ \\
\hline No. of patients & $40(100)$ \\
\hline $\mathrm{M} / \mathrm{F}$ & $1: 1.1$ \\
\hline \multicolumn{2}{|l|}{ Age in yrs } \\
\hline Median & 44 \\
\hline Range & $24-74$ \\
\hline \multicolumn{2}{|l|}{ Localization } \\
\hline Frontal & $13(32)$ \\
\hline Temporal & $13(32)$ \\
\hline Insular & $6(15)$ \\
\hline Parietal & $4(10)$ \\
\hline Limbic & $3(8)$ \\
\hline Central & $1(2)$ \\
\hline \multicolumn{2}{|l|}{ MRI contrast enhancement } \\
\hline Patchy/faint & $16(40)$ \\
\hline None & $13(32)$ \\
\hline Focal & $11(28)$ \\
\hline \multicolumn{2}{|l|}{ Type of surgery } \\
\hline Resection & $36(90)$ \\
\hline Biopsy & $4(10)$ \\
\hline \multicolumn{2}{|l|}{ Diagnosis } \\
\hline \multicolumn{2}{|l|}{ WHO Grade II } \\
\hline Diffuse astrocytoma & $7(18)$ \\
\hline Oligoastrocytoma & $7(18)$ \\
\hline Diffuse oligodendroglioma & $6(15)$ \\
\hline \multicolumn{2}{|l|}{ WHO Grade III } \\
\hline Anaplastic astrocytoma & $11(28)$ \\
\hline Anaplastic oligodendroglioma & $6(15)$ \\
\hline Oligoastrocytoma & $3(8)$ \\
\hline
\end{tabular}

* Values are number of patients (\%) unless stated otherwise.

and faint or focal contrast enhancement) were included; gliomas with significant contrast enhancement (ring-like or nodular contrast enhancement) were excluded as described previously. ${ }^{37-39}$

In the frame of the same MRI investigation, we additionally performed MRS CSI with corresponding specific T2-weighted images and DTI sequences. Furthermore, we obtained T1-weighted sequences without contrast medium administration that were used together with contrast-enhanced T1-weighted images for 3D brain surface and vessel visualization.

\section{Metabolic Imaging}

In the present study the following metabolic imaging modalities were performed in the glioma patients.

PET. Routinely, PET imaging was performed with amino acid tracers using either ${ }^{11} \mathrm{C}$-methionine (MET) or ${ }^{18} \mathrm{~F}$-fluoro-ethyl-L-tyrosine (FET). For both tracers the radiotracer production, data acquisition, and imaging reconstruction were described previously. ${ }^{37,39}$ The intratumoral area with the maximum tumor-to-normal brain ratio $(\mathrm{T} / \mathrm{N} \geq 1.2)$ was visualized as a "hotspot" by using color maps. ${ }^{37,39}$
CSI. If PET was not able to detect an intratumoral area of increased metabolism (T/N < 1.2), multivoxel MRS CSI was used for "hotspot" detection. ${ }^{37}$ Data acquisition and image reconstruction were performed as described previously. ${ }^{38}$ In sum, color-coded CSI maps $(1-\mathrm{cm}$ slice thickness) were created by data postprocessing to visualize the tumor area with the maximum pathological CSI ratio (choline $/ N$-acetylaspartate ratio; pathological ratio $\geq$ 1). For integration of CSI data into the planning station, a specific CSI hybrid data set (15 slices, 2-mm thickness: 5 CSI slices surrounded by 10 empty slices) was produced. Furthermore, specific T2-weighted images (turbo spin echo [TSE] sequence, 15 slices, 2-mm slice thickness) were obtained in the region of the $1-\mathrm{cm}$ CSI slice.

\section{Fiber Tracking}

A DTI sequence was obtained using the following technical MRI data: TR $8000 \mathrm{msec}$, TE $83 \mathrm{msec}$, FOV 256, matrix size $128 \times 128$, diffusion directions 30 , b-values 0 and $800 \mathrm{sec} / \mathrm{mm}$, slice thickness $2 \mathrm{~mm}, 65$ slices, acquisition time 4.34 minutes. For postprocessing of DTI data, we applied the commercially available StealthViz software (Medtronic) that is part of the planning station. This software was used for visualization of the corticospinal tract and the arcuate fascicle. For this purpose, the corticospinal tract was created manually by selecting 2 regions of interest (ROIs) within the cerebral peduncle and the precentral gyrus as defined by anatomical or functional MRI (fMRI) data. For the arcuate fascicle the ROIs were placed within Broca's and Wernicke's area and/or according to the fMRI data.

\section{D Brain Surface Visualization}

For 3D brain surface and vessel visualization, we obtained T1-weighted images without and with contrast medium. Data acquisition and image reconstruction were performed as described previously. ${ }^{15}$

\section{Image Transfer and Fusion}

The structural and metabolic imaging data and MRI sequences for $3 \mathrm{D}$ brain visualization were sent from the picture archiving and communication system into the planning station via the local network. The fiber tract data were exported to the planning station.

For multimodality image fusion, we used the latest Synergy Cranial planning station (Medtronic), with the contrast-enhanced T1-weighted MR image always selected as the reference image. Further structural images, PET scans, and MRI sequences for 3D brain visualization were automatically coregistered with the reference image by the software.

As the single 1-cm CSI slice does not provide enough spatial information for direct image fusion, we performed the following workaround to integrate CSI data in the planning station. Primarily, the specific T2-weighted TSE images (15 slices) were coregistered to the reference image. Subsequently, the CSI hybrid data set (15 slices) was manually linked to the T2-weighted TSE images.

To integrate the fiber tract data, manual coregistration of these data to the reference image was necessary, as the anatomical imaging data for tractography were equivalent to the reference image. 


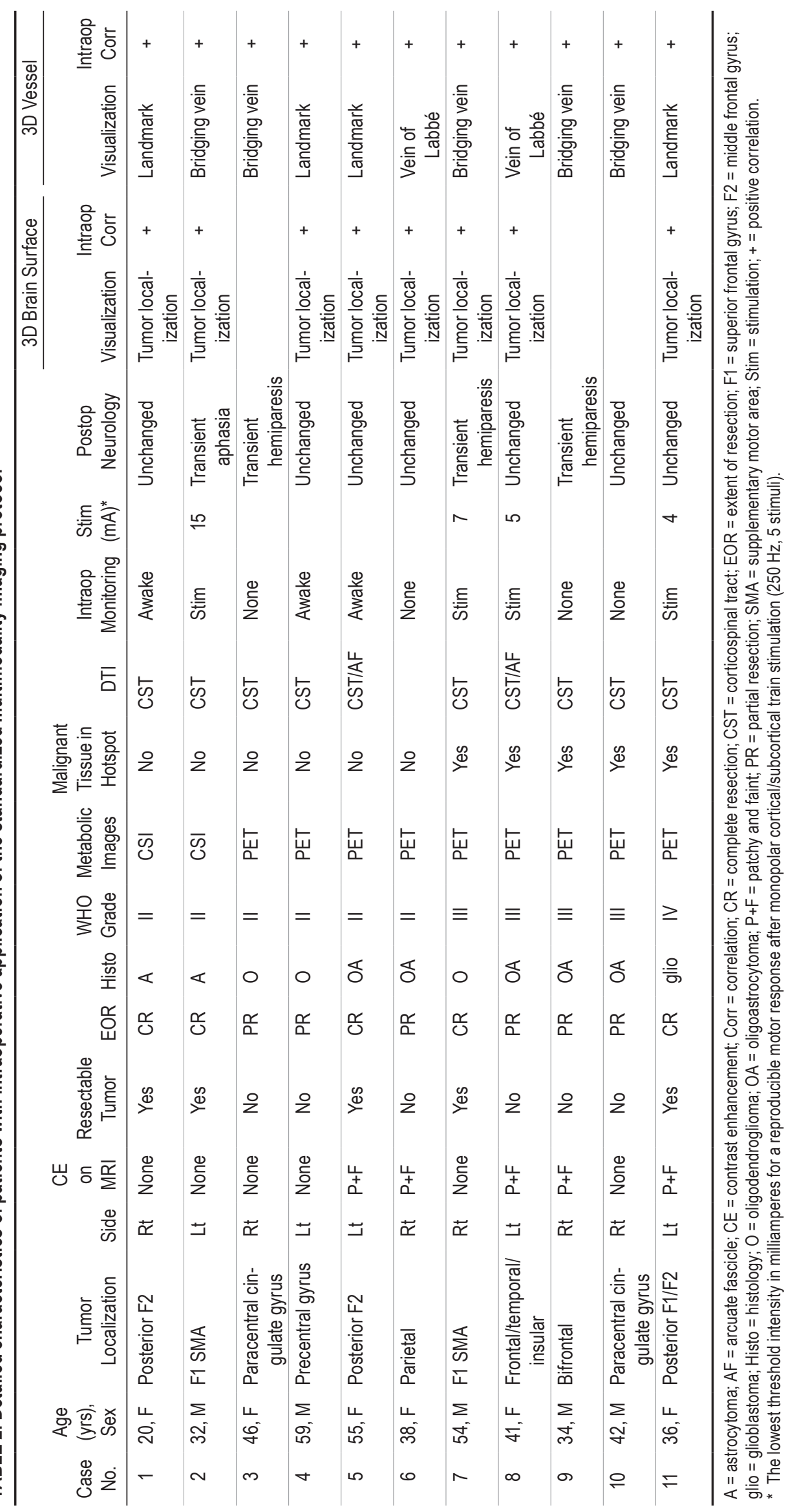




\section{Definition of Standardized Image Settings}

After image fusion, we performed a retrospective review of the coregistered multiple image data obtained in the 40 glioma patients to define standardized examination settings. The following planning station tools are essential for image setting adjustments: 1) Image modality and color map. By selecting an image modality such as MRI, CT, PET, or fMRI the specific examination is assigned to this modality and thus a preset color map is automatically applied (e.g., selecting "PET" within the image modality tool results in a rainbow-colored examination). 2) Level and width. By changing the level and width settings, adjustments in brightness and radiographic contrast are performed. 3) Transparency. This function enables modifications of the transparency of a selected examination. While a transparency of $100 \%$ means that the selected examination is fully visible, decreasing the transparency to $0 \%$ results in complete disappearance of the examination. 4) 3D objects and models tool. Tumor objects and brain surface models can be visualized in $2 \mathrm{D}$ or $3 \mathrm{D}$ with this tool. Within this tool the anatomical examination (e.g., T2weighted and T1-weighted MRI) and the intended object such as "tumor" or "brain" have to be selected. Then either manual segmentation using basic drawing tools or a semiautomatic segmentation using thresholds can be performed to create a new object (tumor) or a new 3D brain model. For 3D visualization of brain models a "basic" or "shaded" color map can be selected. 5) 3D model import. Another feature of the 3D model tool is called "import models" which imports fiber tracts or objects created with the StealthViz software.

\section{Assessment of the Different Imaging Modalities}

In a further step, the impact of these different multiple imaging modalities was assessed by 2 experienced neurosurgeons (S.W. and G.W.) as follows: 1) impact of T2-weighted or FLAIR images for delineation of tumor borders; 2) impact of metabolic imaging (PET or CSI) for identification of an intratumoral hotspot; 3 ) impact of DTI for preoperative visualization of important white matter tracts (corticospinal tract and arcuate fascicle) adjacent to gliomas; and 4) impact of 3D brain surface visualization for precise tumor localization and 3D brain vessel visualization for approach planning, intraoperative orientation and identification of bridging veins, vein of Labbé, and specific cortical vessels.

\section{Intraoperative Application of the Standardized Multimodality Imaging Protocol}

Both the results of the assessment of the imaging modalities and the standardized image settings defined our protocol for the multiple imaging modalities. Its feasibility and surgical relevance was then prospectively analyzed during glioma surgery with the assistance of an advanced electromagnetic navigation system (S7 AxiEM, Medtronic) in the remaining 11 patients. ${ }^{15}$ Additionally, we assessed the following surgical outcome parameters in these glioma patients to clinically validate each image modality of our proposed protocol: 1) Extent of resection. The extent of resection was analyzed on postoperative MRI that was conducted within 72 hours after surgery and was classified according to the RANO criteria (complete or partial resection). ${ }^{36}$ The extent of resection was of special interest in gliomas that were considered to be resectable according to tumor localization on preoperative imaging. 2) Histological analysis of the metabolic hotspot. Tissue sampling was performed from the metabolic hotspot (PET or CSI) by navigational guidance as described previously, and the collected tumor specimens were screened for the presence of malignant tissue according to the current histopathological WHO criteria. ${ }^{13,37-39}$ 3) Presence of a new postoperative neurological deficit. In each patient, the preoperative as well as the postoperative neurological status was investigated to detect a new neurological deficit (transient or permanent neurological deficit) after glioma surgery. A permanent neurological deficit was present if the new postoperative neurological deficit persisted until the first follow-up examination 3 months after surgery. 4) Analysis of the accuracy of 3D brain visualization. Intraoperative topographical correlation of the 3D brain surface and vessel models with gyral anatomy and superficial vessels was performed as described previously. ${ }^{14}$

\section{Results}

In the present study, altogether 51 gliomas with nonsignificant contrast enhancement on MRI were included to define a standardized multimodality imaging protocol (n $=40$ ) that was subsequently prospectively validated during advanced navigation-guided glioma surgery $(\mathrm{n}=11)$.

\section{Definition of Standardized Image Settings}

Initially, we integrated the multiple imaging data obtained from the first 40 glioma patients into our planning station and retrospectively reviewed these data to define standardized settings of structural, metabolic, and fiber tract data and 3D brain visualization. We aimed at enabling an optimal concurrent visualization of all relevant multimodality image data in a single picture. Details of the standardized imaging settings for each modality are provided in Table 3.

\section{Structural Imaging}

T1-Weighted Images With Contrast Medium, T2Weighted, and FLAIR Sequences. The planning station recognizes structural MR images and automatically selects the "grayscale" color map resulting in predefined level and width settings. Additional adjustments can be made depending on the intended visualization of the tumor and surrounding white and gray matter.

Contour Visualization. The planning station is capable of outlining only the contour of specific pathological or functional objects. For contour visualization of tumor borders, we propose selecting T2-weighted or FLAIR images to create a specific "object" by using the 3D model tool. Subsequently, the contour of this object is then superimposed on the reference study (T1-weighted contrast-enhanced MRI), enabling the concurrent visualization of the exact tumor border and all additional coregistered image data. 
TABLE 3. Standardized image settings of the different imaging modalities

\begin{tabular}{|c|c|c|c|c|c|c|c|}
\hline Variable & Imaging Sequences & $\begin{array}{l}\text { Imaging } \\
\text { Modality }\end{array}$ & Color Map & Level & Width & Transparency & $\begin{array}{c}\text { Contour } \\
\text { Visualizatior }\end{array}$ \\
\hline \multirow[t]{2}{*}{ Structural imaging } & $\begin{array}{l}\text { T1-weighted MRI w/ } \\
\quad \text { CM }\end{array}$ & MRI & Grayscale & System present & System present & & \\
\hline & $\begin{array}{l}\text { T2-weighted MRI or } \\
\text { FLAIR }\end{array}$ & MRI & Grayscale & System present & System present & & 2D mode \\
\hline \multirow[t]{2}{*}{ Metabolic imaging } & PET & PET & Rainbow & $25,000 \pm 1000$ & $6000 \pm 1000$ & $20 \%$ & \\
\hline & CSI & SPECT & Rainbow & Maximal & $150 \pm 100$ & $20 \%$ & \\
\hline Fiber tracking & DTI & & & & & & 2D mode \\
\hline 3D brain visualization & $\begin{array}{l}\text { T1-weighted MRI } \\
\text { w/o \&/or w/ CM }\end{array}$ & & Basic or shaded & $\begin{array}{l}\text { Lower threshold } \\
50-300\end{array}$ & $\begin{array}{l}\text { Upper threshold } 1200 \\
\text { filter medium }\end{array}$ & & \\
\hline
\end{tabular}

$\mathrm{CM}=$ contrast medium .

\section{Metabolic Imaging}

$P E T$. As the PET scan is not automatically recognized by the planning station, the first step is to define it as "PET" in the image modality, which leads to the "rainbow" mode in the color map and thus enables color-coded visualization of the PET data (Fig. 1). To highlight only the intratumoral area with the highest metabolic activity corresponding to the PET hotspot, we propose to increase the level value to $25,000 \pm 1000$ and adjust the width value to $6000 \pm 1000$. Furthermore, for a simultaneous visualization of the PET hotspot with the structural images in the background, it was found to be optimal to decrease the transparency of the PET scan from $100 \%$ to $20 \%$.
CSI. This time, "SPECT" has to be selected within the image modality to allow a "rainbow" color-coded visualization of the CSI data (Fig. 1). The optimal adjustments for CSI data were defined as follows: the level value has to be increased to maximum and the width value should be adjusted to $150 \pm 100$ and the transparency to $20 \%$.

\section{Fiber Tracking}

After image fusion, the specific objects created on the planning station were automatically extracted out of the fiber tract data by using the 3D model and import models tool. The tracts were visualized as 3D fiber bundles within the 3D brain surface model or as 2D fiber contours (contour visualization) superimposed on the reference image.
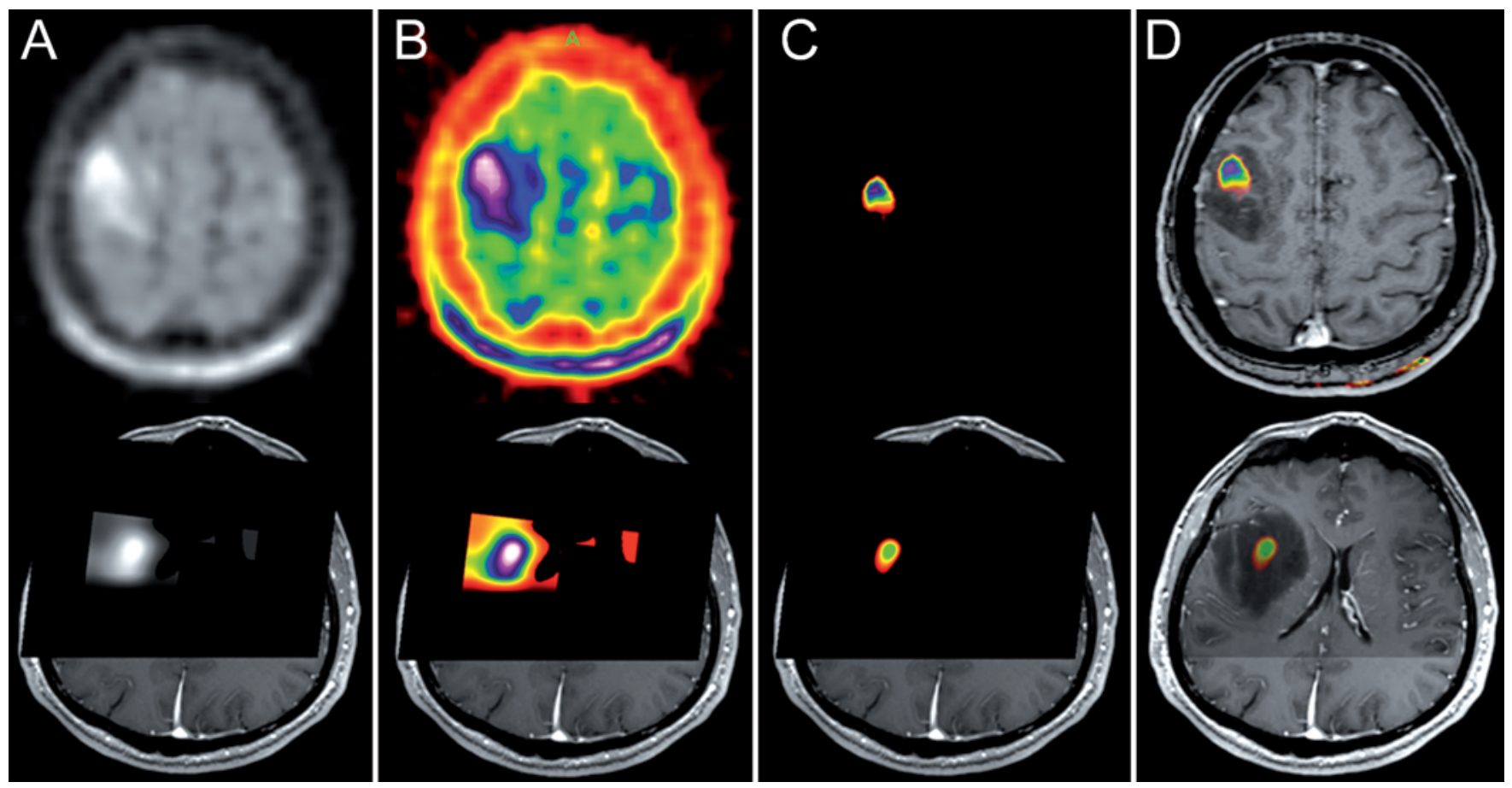

FIG. 1. Illustration of the standardized image settings in metabolic images using PET and MRS CSI. PET (upper) and CSI images (lower) on the planning station. A and B: The raw data of the PET scan and the specific CSI hybrid data set both in black and white (A) are converted into color-coded images (B). C: By specific adjustment of the level and width value, only the intratumoral PET and CSI hotspot can be visualized. D: Decreasing the transparency to $20 \%$ allows a concurrent visualization of structural images using contrast-enhanced T1-weighted images and the PET and CSI hotspot for navigation-guided tissue sampling. 


\section{D Brain Visualization}

An anatomical 3D model of the brain can be created using the 3D model tool of the planning station. After selection of the T1-weighted images without contrast medium the lower threshold has to be increased by stages until only the brain tissue is visible and the surrounding bone, fat, and skin disappears (lower threshold 50-300 and upper threshold 1200). To obtain a detailed 3D brain model with detailed gyral and sulcal structures, we prefer the "basic" mode in the color map within the 3D model display. In contrast, for 3D brain vessel visualization we apply T1weighted images with contrast medium that enable 3D brain models with visualization of superficial vascular structures. For an optimal visualization of the 3D vascular pattern either the "basic" or "shaded" mode in the color map can be selected. A concurrent visualization of both $3 \mathrm{D}$ brain models is possible.

\section{Analysis of Impact of the Different Imaging Modalities}

Second, the expected benefit of the various image modalities was retrospectively analyzed in each of the 40 cases by 2 experienced neurosurgeons (S.W. and G.W.) to define specific indications for the different imaging modalities. For each imaging modality, we applied the standardized imaging settings described above. A detailed overview of these indications is provided in Table 4, and the multimodality image data are illustrated in Fig. 2. 1) Impact of T2weighted or FLAIR images for delineation of the tumor borders. The application of T2-weighted or FLAIR images in the context of the contour visualization function was considered useful in all cases ( $\mathrm{n}=36 ; 90 \%$ of cases) that are treated by resection for precise definition of the tumor margin. 2) Impact of metabolic imaging (PET or CSI) for identification of an intratumoral hotspot. The application of metabolic imaging using PET or CSI was considered useful in all cases; PET was capable of identifying an intratumoral hotspot $(\mathrm{T} / \mathrm{N}$ ratio $\geq 1.2)$ in 33 patients. CSI was able to detect a CSI hotspot in all cases of a negative PET (T/N ratio < 1.2; $\mathrm{n}=7$ ). 3) Impact of fiber tracking for visualization of relevant white matter tracts adjacent to gliomas. Forty percent of the gliomas $(\mathrm{n}=16)$ were localized close to eloquent cortical or subcortical structures (motor or speech area). Consequently, fiber tracking was considered useful in these patients, especially to limit glioma resection and thus preserve important functional structures. 4) Impact of 3D brain visualization. 3D brain surface visualization was considered useful for precise localization of all gliomas (n $=20$ patients; $50 \%$ of cases) situated at the brain convexity. In contrast, only minor benefit of 3D brain surface visualization was expected in cases of deep-seated tumors and repeated surgeries.

The 3D brain vessel visualization was deemed beneficial for approach planning and intraoperative orientation in 30 patients (75\% of cases). To this end, especially the identification of bridging veins, vein of Labbé, and specific cortical vessels that serve as anatomical landmarks was considered useful.

\section{Intraoperative Application of the Standardized Multimodality Imaging Protocol}

The standardized imaging settings together with the specific indications for each imaging modality defined our new multimodality imaging protocol for gliomas with nonsignificant contrast enhancement (Tables 3 and 4). This newly established protocol including structural images, metabolic data, fiber tracking, and/or 3D surface visualization was then prospectively applied during navigationguided surgery in 11 glioma patients (Table 2). Intraoperative monitoring with either cortical/subcortical stimulation $(n=4)$ or awake surgery $(n=3)$ was additionally applied to preserve neurological function if indicated. According to the appraisal of the neurosurgeons performing the surgery, this protocol was feasible and was rated surgically relevant in all cases. By using this protocol all surgically important imaging modalities were concurrently visualized in each case. In all gliomas that were considered to be resectable according to preoperative imaging $(n=5)$, a complete tumor resection was achieved by using contour visualization with T2-weighted or FLAIR images. Moreover, all tumor samples from the metabolic hotspot (PET or CSI) of each WHO Grade III or IV glioma $(n=5)$ revealed malignant tissue according to the histopathological WHO criteria. Additionally, the use of fiber tracking was a powerful tool to intraoperatively define brain areas adjacent to the glioma in which relevant white matter tracts are expected. The application of intraoperative monitoring such as cortical/subcortical stimulation or awake surgery provided the neurosurgeon with additional "real-time" in-

TABLE 4. Specific indications of the different imaging modalities

\begin{tabular}{llll}
\hline \multicolumn{1}{c}{ Variable } & \multicolumn{1}{c}{ Image Sequences } & Specific Indication & Intraop Value \\
\hline Structural imaging & T1-weighted MRI w/ CM & All suspected LGGs & Reference study for navigation \\
\hline & T2-weighted MRI or FLAIR & All suspected LGGs w/ tumor resection & $\begin{array}{c}\text { Definition of glioma border (contour } \\
\text { visualization) }\end{array}$ \\
\hline Metabolic imaging & PET & All suspected LGGs & Detection of an intratumoral hotspot \\
\hline Fiber tracking & CSI & Suspected LGGs w/ negative PET & Detection of an intratumoral hotspot \\
\hline 3D brain visualization & T1-weighted MRI & $\begin{array}{c}\text { Suspected LGGs close to eloquent cortical or subcortical } \\
\text { structures (motor or speech area) }\end{array}$ & $\begin{array}{c}\text { Limitation of glioma resection } \\
\end{array}$ \\
& T1-weighted MRI w/ CM & $\begin{array}{c}\text { Suspected LGGs localized at the brain convexity } \\
\text { vein of Labbé, \& specific cortical vessels is crucial }\end{array}$ & $\begin{array}{c}\text { Precise localization of gliomas (3D brain } \\
\text { surface visualization) }\end{array}$ \\
\hline
\end{tabular}



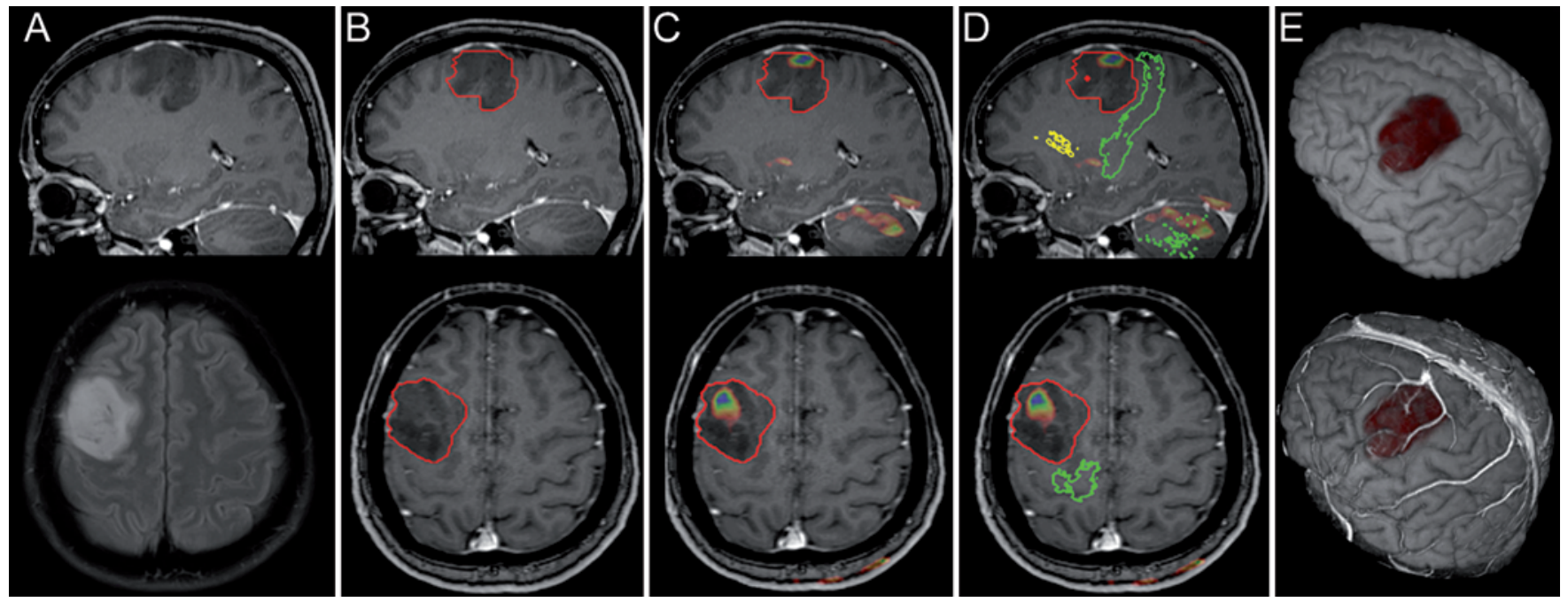

FIG. 2. Illustration of the multimodality imaging data on the planning station that were obtained in a patient with a suspected LGG. A: Structural images consisting of contrast-enhanced T1-weighted images (reference image for navigation, upper) and FLAIR sequence (lower) reveal a lesion in the left frontal premotor region. B: The tumor volume was segmented using FLAIR images and is superimposed as red contour on the reference image. C: Furthermore, the color-coded PET "hotspot" is added. D: In the next step, fiber tracking data consisting of the corticospinal tract (green contour) and the arcuate fascicle (yellow contour) are included. E: Finally, the 3D brain surface model (upper) based on T1-weighted images without contrast medium administration clearly shows the exact topographic localization of the tumor (red) in the middle frontal gyrus. The 3D brain vessel model (lower) based on additional contrast-enhanced T1-weighted images enables visualization of the vascular brain surface anatomy important for approach planning and intraoperative orientation.

formation that is unaffected by brain shift regarding the integrity of the language/motor fiber tracts during tumor removal. A permanent postoperative neurological deficit was not observed in any of these 11 patients (unchanged neurological findings in 7 cases; transient neurological deficit in 4 cases), whereby DTI (10 cases) and/or intraoperative monitoring ( 7 cases) were applied during surgery in the vast majority of patients. Finally, the 3D brain surface and vessel models showed a significant intraoperative topographical correlation with gyral anatomy and superficial vessels in all cases in which this imaging technique was considered to be useful. Further details are provided in Table 2.

The additional application of electromagnetic instrument tracking enabled continuous navigation guidance during glioma surgery. For this purpose, a flexible "stylet" integrated in the suction device allowed "real-time" navigation using the different imaging modalities. This technique was especially helpful for precise guidance to surgical targets such as the intratumoral metabolic "hotspot" or the tumor margin. Two representative cases (Cases 4 and 11) of the 11 glioma patients are illustrated in Figs. 3 and 4.

\section{Discussion}

In the present study, we defined a new multimodality imaging protocol for advanced navigation-guided surgery of diffusely infiltrating gliomas with nonsignificant contrast enhancement on MRI. To this end, we developed standardized imaging settings and specific indications for structural and metabolic data, fiber tracking, and 3D brain visualization. The prospective clinical validation of this new protocol during navigation-guided surgery showed that it was feasible and surgically relevant in all cases and provided substantial support for the neurosurgeon to achieve the essential goals of modern glioma surgery.

\section{Neuronavigation in Glioma Surgery}

To optimize surgery of diffusely infiltrating gliomas, several advancements such as intraoperative MRI, fluorescence-guided procedures, brain mapping, and neurophysiological stimulation have been implemented in the last few decades. ${ }^{6,27,31,37,39}$ One of the most important improvements was the introduction of the cranial neuronavigation by Roberts et al. almost 30 years ago. ${ }^{23}$ Neuronavigation systems allow improved preoperative planning to facilitate minimally invasive neurosurgical approaches and exact intraoperative localization of gliomas and surrounding brain structures. ${ }^{15,34}$ Consequently, neuronavigation systems are nowadays considered standard in glioma surgery. Recently, advanced navigation systems that allow continuous neurosurgical instrument tracking with the aid of electromagnetic technology were introduced for glioma surgery. ${ }^{15}$

\section{Neuronavigation With Multimodal Imaging Techniques}

In routine clinical practice, navigation guidance during tumor surgery is based mainly on structural MRI data to outline the tumor margins in relation to the adjacent brain anatomy. ${ }^{12,40}$ In the past few years, advanced neuronavigation systems that are capable of additionally integrating and visualizing multimodality image data have evolved. ${ }^{18}$ First of all, navigation with metabolic image data derived from PET or CSI represents a powerful tool to identify the metabolically most active intratumoral areas to detect anaplastic foci during glioma surgery. ${ }^{20,30,34,38}$ Furthermore, navigation with DTI has been increasingly applied 

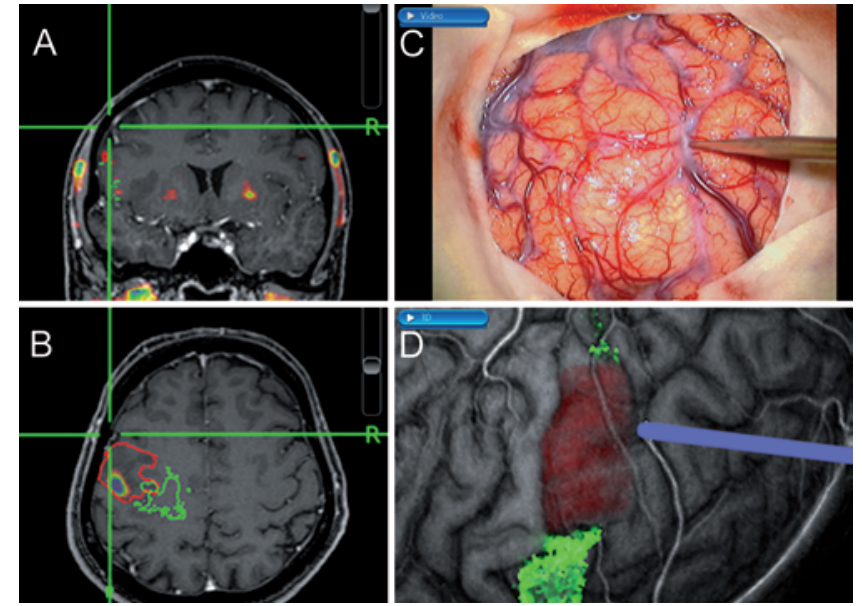

FIG. 3. Case 4. Intraoperative navigation using the multimodality imaging protocol in a patient with a suspected LGG. A and B: During glioma resection, navigation with concurrent display of contour visualization of the tumor border (red contour), the metabolic hotspot using PET (color-coded region), and fiber tracking data with the corticospinal tract (green contour) was performed. C and D: Intraoperative navigation with continuous instrument tracking shows an excellent correlation of the brain surface and vessel anatomy $(\mathrm{C})$ and the corresponding 3D brain model (D) that supports precise lesion localization and intraoperative orientation.

to visualize important white matter tracts adjacent to gliomas to limit tumor resection and reduce the risk of a new postoperative neurological deficit. ${ }^{1,5,17}$ Recently, navigation with 3D brain visualization was proposed for precise localization of the glioma anatomy and vasculature. ${ }^{10,14} \mathrm{Al}-$ though these different multimodality imaging data can be simultaneously displayed on one navigation screen, still no standardized protocol for navigation with multimodality image data of suspected LGGs that fulfills the specific requirements of modern glioma surgery exists.

\section{Current Study-Proposed Multimodality Image Protocol}

Herein, we defined such a standardized protocol for advanced navigation with multimodality imaging data in diffusely infiltrating gliomas with nonsignificant contrast enhancement on MRI. This new protocol included only selected imaging modalities that resulted in a distinct improvement in glioma surgery in prior clinical studies. . $^{1,12,14,17,20,30,34,38,40}$ We first performed a retrospective review of specific multimodality imaging data of 40 patients with suspected LGG on our planning station. To this end, we were able to define standardized imaging settings of structural and metabolic data, fiber tracking data, and 3D brain visualization that allow optimal and simultaneous visualization of the multimodality imaging data (Table 3).

In the next step, we analyzed the expected impact of the multimodality imaging data obtained in the 40 glioma patients to define specific indications for each image modality. Based on these findings, we determined the following indications for gliomas with nonsignificant contrast enhancement on MRI (Table 4): 1) Structural images using T2-weighted or FLAIR sequences are indicated especially for visualization of the glioma margin via the contour visualization function of the planning station in all suspected LGGs treated by resection. 2) Metabolic images using PET or CSI data are indicated for detection of potential intratumoral hotspots in all suspected LGGs. In the few cases in which PET or CSI is not capable of identifying a distinct intratumoral hotspot, our concept is to collect multiple tissue samples of glioma areas with different macroscopic appearance and consistency or to perform tissue sampling from an intratumoral area with focal contrast
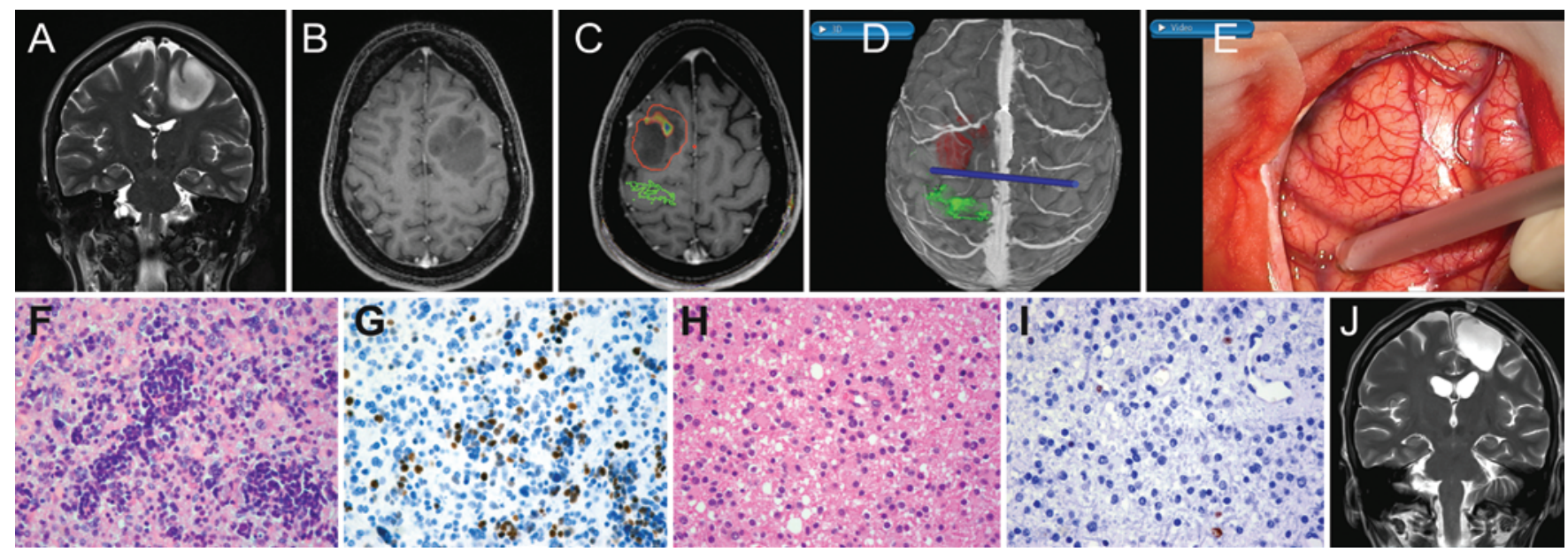

FIG. 4. Case 11. Intraoperative application of the multimodality imaging protocol in a patient with a glioma with nonsignificant contrast enhancement. A and B: Preoperative MR images revealing a left frontal hyperintense lesion on T2-weighted images (A) with patchy and faint contrast enhancement on contrast-enhanced T1-weighted sequences (B). C: During the glioma resection, navigation with multimodality imaging data including contour visualization of the tumor border (red contour), the metabolic PET hotspot (color-coded region), and the corticospinal tract (green contour) was conducted. D and E: Additionally, electromagnetic navigation with a 3D brain surface and vessel visualization model was performed (D) that showed a significant correlation with the brain surface and vessel anatomy (E). F-I: Histopathological examination of the tumor sample derived from the PET hotspot depicts WHO Grade IV glioma tissue (F) with a high proliferation index $(\mathrm{G})$, whereas the tumor specimen from outside the metabolic hotspot shows only WHO Grade II glioma tissue with a low proliferation rate (I). H \& E (F and H) and anti-Ki 67 (G and I), original magnification $\times 200$. J: Postoperative T2-weighted MR image demonstrating complete resection of the lesion. 
enhancement on MRI. ${ }^{37,39}$ 3) Fiber tracking is indicated in suspected LGGs localized close to eloquent cortical or subcortical structures such as motor or speech areas to limit tumor resection. 4) 3D brain surface visualization is indicated for precise tumor localization especially in gliomas that are localized at the brain convexity. Furthermore, $3 \mathrm{D}$ brain vessel visualization is indicated in the majority of suspected LGGs for approach planning and intraoperative orientation and in gliomas in which the intraoperative identification of anatomical landmarks such as bridging veins, vein of Labbé or specific cortical vessels is crucial. These specific indications together with the standardized imaging settings defined our new protocol for navigation with multimodality imaging data.

\section{Intraoperative Application of the Multimodality Imaging Protocol}

Finally, this new multimodality imaging protocol was prospectively clinically validated during advanced navigation-guided surgery in 11 patients with a diffusely infiltrating glioma with nonsignificant contrast enhancement on MRI (Table 2). For this purpose, we applied a latest generation navigation system that allows continuous tracking of neurosurgical instruments, such as the suction device, by using electromagnetic technology. In visualizing all surgically important imaging modalities, this new protocol was feasible and was estimated to be surgically relevant during navigation-guided glioma surgery in all 11 cases. The advantage of our new navigation protocol is that the surgically relevant information concurrently displayed is reduced to the least amount possible that still contains all necessary data for optimal glioma surgery. Overwhelming the neurosurgeon with too much information can thus be prevented.

According to our predefined surgical outcome parameters, we found that in all resectable gliomas a complete resection was achieved using contour visualization with structural images. Additionally, histopathological examination of tumor tissue derived from the metabolic hotspot (PET or CSI) demonstrated the presence of malignant tissue according to the WHO criteria in all WHO Grade III or IV gliomas. Moreover, a permanent postoperative neurological deficit was observed in none of our patients, whereby DTI and/or intraoperative monitoring were applied in the vast majority of cases. Furthermore, we found a significant intraoperative topographical correlation of 3D brain surface and vessel models with gyral anatomy and superficial vessels. Finally, electromagnetic real-time navigation with multimodality imaging data was a helpful tool for precise guidance to surgical targets, such as intratumoral metabolic hotspots or the tumor margin in our glioma patients. In this sense, Suess et al. described the successful application of electromagnetic real-time navigation for surgery of especially deep-seated brain lesions. ${ }^{33}$ Moreover, our group applied electromagnetic navigation with continuous instrument tracking during surgery of 71 brain tumors and found that this technique was particularly useful in combination with DTI to limit tumor resection. ${ }^{15}$ Recently, Harrisson et al. recommended the application of frameless electromagnetic navigation as an effective technique for biopsies of different intracranial lesions. ${ }^{8}$ According to these data in the literature, including our current study, we propose the use of electromagnetic navigation in conjunction with our new multimodality imaging protocol. We expect that the application of this multimodality imaging protocol will optimize surgery of suspected LGG and thus improve the patient prognosis and outcome of these tumors in the future.

\section{Limitations of the Study}

Some limitations of our study have to be addressed. First of all, we did not include fMRI data in our multimodality imaging protocol. However, fMRI data were used for definition of ROIs in the frame of creation of fiber tracts. Furthermore, we performed a retrospective analysis with its known disadvantages of the expected benefit of the multimodality imaging data of the first 40 patients. Nevertheless, our new protocol was subsequently prospectively applied and clinically validated during navigation-guided surgery in 11 patients. Additionally, brain shift causes increasing inaccuracy of the navigation system with the integrated multimodality imaging data during glioma surgery, and thus neuronavigation is accurate only at the beginning of surgery. ${ }^{16,22}$ However, the 3D brain surface and vessel visualization models remain applicable even after dura opening and loss of cerebrospinal fluid, since the topographic relation between the brain surface anatomy and vessels is unaffected by brain shift. ${ }^{14}$ Moreover, accurate tissue sampling from the metabolic hotspot can be confirmed independent of brain shift with the additional use of fluorescence guidance with 5-aminolevulinic acid. ${ }^{37,39}$ Finally, in our department we use a planning station and navigation system manufactured by Medtronic that served as the basis for the present study to develop the standardized protocol for multimodality imaging data. However, integration of such multiple imaging data into neuronavigation can be performed with other systems on the market as well.

\section{Conclusions}

The present study defines a standardized protocol for advanced navigation with multimodality imaging data in patients with suspected LGGs. According to our data, the establishment of standardized imaging settings and specific indications allowed an optimal and simultaneous visualization of structural and metabolic data, fiber tracking, and 3D brain surface and vessel visualization. Subsequent prospective intraoperative application of this new protocol demonstrated that it was feasible, surgically relevant, and provided substantial support for the neurosurgeon to achieve the essential goals of modern glioma surgery. Therefore, we propose this multimodality imaging protocol for advanced navigation-guided procedures optimally in conjunction with continuous electromagnetic instrument tracking to optimize surgical treatment of suspected LGGs.

\section{References}

1. Abdullah KG, Lubelski D, Nucifora PGP, Brem S: Use of diffusion tensor imaging in glioma resection. Neurosurg Focus 34(4):E1, 2013

2. Burger PC, Scheithauer BW, Vogel FS: Surgical Pathology of the Nervous System and Its Coverings, ed 3. New York: Churchill Livingstone, 1991 
3. Capelle L, Fontaine D, Mandonnet E, Taillandier L, Golmard JL, Bauchet L, et al: Spontaneous and therapeutic prognostic factors in adult hemispheric World Health Organization Grade II gliomas: a series of 1097 cases. Clinical article. J Neurosurg 118:1157-1168, 2013

4. Cha S: Neuroimaging in neuro-oncology. Neurotherapeutics 6:465-477, 2009

5. Coenen VA, Krings T, Mayfrank L, Polin RS, Reinges MH, Thron A, et al: Three-dimensional visualization of the pyramidal tract in a neuronavigation system during brain tumor surgery: first experiences and technical note. Neurosurgery 49:86-93, 2001

6. De Witt Hamer PC, Robles SG, Zwinderman AH, Duffau H, Berger MS: Impact of intraoperative stimulation brain mapping on glioma surgery outcome: a meta-analysis. J Clin Oncol 30:2559-2565, 2012

7. Duffau H, Capelle L: Preferential brain locations of lowgrade gliomas. Cancer 100:2622-2626, 2004

8. Harrisson SE, Shooman D, Grundy PL: A prospective study of the safety and efficacy of frameless, pinless electromagnetic image-guided biopsy of cerebral lesions. Neurosurgery 70:29-33, 2012

9. Hervey-Jumper SL, Berger MS: Role of surgical resection in low- and high-grade gliomas. Curr Treat Options Neurol 16:284, 2014

10. Kockro RA, Reisch R, Serra L, Goh LC, Lee E, Stadie AT: Image-guided neurosurgery with 3-dimensional multimodal imaging data on a stereoscopic monitor. Neurosurgery 72 (Suppl 1):78-88, 2013

11. Kunz M, Thon N, Eigenbrod S, Hartmann C, Egensperger R, Herms J, et al: Hot spots in dynamic (18)FET-PET delineate malignant tumor parts within suspected WHO grade II gliomas. Neuro Oncol 13:307-316, 2011

12. Kurimoto M, Hayashi N, Kamiyama H, Nagai S, Shibata T, Asahi T, et al: Impact of neuronavigation and image-guided extensive resection for adult patients with supratentorial malignant astrocytomas: a single-institution retrospective study. Minim Invasive Neurosurg 47:278-283, 2004

13. Louis DN, Ohgaki H, Wiestler OD, Cavenee WK (eds): WHO Classification of Tumours of the Central Nervous System. Lyon: IARC Press, 2007

14. Mert A, Buehler K, Sutherland GR, Tomanek B, Widhalm G, Kasprian G, et al: Brain tumor surgery with 3-dimensional surface navigation. Neurosurgery 71:ons286-ons295, 2012

15. Mert A, Gan LS, Knosp E, Sutherland GR, Wolfsberger S: Advanced cranial navigation. Neurosurgery 72 (Suppl 1):43-53, 2013

16. Nimsky C, Ganslandt O, Cerny S, Hastreiter P, Greiner G, Fahlbusch R: Quantification of, visualization of, and compensation for brain shift using intraoperative magnetic resonance imaging. Neurosurgery 47:1070-1080, 2000

17. Nimsky C, Ganslandt O, Fahlbusch R: Implementation of fiber tract navigation. Neurosurgery 58 (4 Suppl 2):ONS292-ONS-304, 2006

18. Nimsky C, Kuhnt D, Ganslandt O, Buchfelder M: Multimodal navigation integrated with imaging. Acta Neurochir Suppl 109:207-214, 2011

19. Paulus W, Peiffer J: Intratumoral histologic heterogeneity of gliomas. A quantitative study. Cancer 64:442-447, 1989

20. Pirotte B, Goldman S, Dewitte O, Massager N, Wikler D, Lefranc F, et al: Integrated positron emission tomography and magnetic resonance imaging-guided resection of brain tumors: a report of 103 consecutive procedures. J Neurosurg 104:238-253, 2006

21. Preusser M, de Ribaupierre S, Wöhrer A, Erridge SC, Hegi M, Weller M, et al: Current concepts and management of glioblastoma. Ann Neurol 70:9-21, 2011

22. Roberts DW, Hartov A, Kennedy FE, Miga MI, Paulsen KD:
Intraoperative brain shift and deformation: a quantitative analysis of cortical displacement in 28 cases. Neurosurgery 43:749-760, 1998

23. Roberts DW, Strohbehn JW, Hatch JF, Murray W, Kettenberger H: A frameless stereotaxic integration of computerized tomographic imaging and the operating microscope. J Neurosurg 65:545-549, 1986

24. Sanai N, Berger MS: Glioma extent of resection and its impact on patient outcome. Neurosurgery 62:753-764, 2008

25. Sanai N, Snyder LA, Honea NJ, Coons SW, Eschbacher JM, Smith KA, et al: Intraoperative confocal microscopy in the visualization of 5-aminolevulinic acid fluorescence in lowgrade gliomas. J Neurosurg 115:740-748, 2011

26. Scherer HJ: Cerebral astrocytomas and their derivatives. Am J Cancer 40:343-354, 1940

27. Senft C, Bink A, Franz K, Vatter H, Gasser T, Seifert V: Intraoperative MRI guidance and extent of resection in glioma surgery: a randomised, controlled trial. Lancet Oncol 12:997-1003, 2011

28. Smith JS, Chang EF, Lamborn KR, Chang SM, Prados MD, Cha $\mathrm{S}$, et al: Role of extent of resection in the long-term outcome of low-grade hemispheric gliomas. J Clin Oncol 26:1338-1345, 2008

29. Soffietti R, Baumert BG, Bello L, von Deimling A, Duffau $\mathrm{H}$, Frénay M, et al: Guidelines on management of low-grade gliomas: report of an EFNS-EANO Task Force. Eur J Neurol 17:1124-1133, 2010

30. Stadlbauer A, Moser E, Gruber S, Nimsky C, Fahlbusch R, Ganslandt O: Integration of biochemical images of a tumor into frameless stereotaxy achieved using a magnetic resonance imaging/magnetic resonance spectroscopy hybrid data set. J Neurosurg 101:287-294, 2004

31. Stummer W, Pichlmeier U, Meinel T, Wiestler OD, Zanella F, Reulen HJ: Fluorescence-guided surgery with 5-aminolevulinic acid for resection of malignant glioma: a randomised controlled multicentre phase III trial. Lancet Oncol 7:392401, 2006

32. Stupp R, Mason WP, van den Bent MJ, Weller M, Fisher B, Taphoorn MJB, et al: Radiotherapy plus concomitant and adjuvant temozolomide for glioblastoma. N Engl J Med 352:987-996, 2005

33. Suess O, Kombos T, Kurth R, Suess S, Mularski S, Hammersen S, et al: Intracranial image-guided neurosurgery: experience with a new electromagnetic navigation system. Acta Neurochir (Wien) 143:927-934, 2001

34. Tanaka Y, Nariai T, Momose T, Aoyagi M, Maehara T, Tomori $\mathrm{T}$, et al: Glioma surgery using a multimodal navigation system with integrated metabolic images. J Neurosurg 110: 163-172, 2009

35. van den Bent MJ, Wefel JS, Schiff D, Taphoorn MJB, Jaeckle $\mathrm{K}$, Junck L, et al: Response assessment in neuro-oncology (a report of the RANO group): assessment of outcome in trials of diffuse low-grade gliomas. Lancet Oncol 12:583-593, 2011

36. Vogelbaum MA, Jost S, Aghi MK, Heimberger AB, Sampson JH, Wen PY, et al: Application of novel response/progression measures for surgically delivered therapies for gliomas: Response Assessment in Neuro-Oncology (RANO) Working Group. Neurosurgery 70:234-244, 2012

37. Widhalm G, Kiesel B, Woehrer A, Traub-Weidinger T, Preusser M, Marosi C, et al: 5-Aminolevulinic acid induced fluorescence is a powerful intraoperative marker for precise histopathological grading of gliomas with non-significant contrast-enhancement. PLoS ONE 8:e76988, 2013

38. Widhalm G, Krssak M, Minchev G, Wöhrer A, TraubWeidinger T, Czech T, et al: Value of $1 \mathrm{H}$-magnetic resonance spectroscopy chemical shift imaging for detection of anaplastic foci in diffusely infiltrating gliomas with non-significant 
contrast-enhancement. J Neurol Neurosurg Psychiatry 82:512-520, 2011

39. Widhalm G, Wolfsberger S, Minchev G, Woehrer A, Krssak M, Czech T, et al: 5-Aminolevulinic acid is a promising marker for detection of anaplastic foci in diffusely infiltrating gliomas with nonsignificant contrast enhancement. Cancer 116:1545-1552, 2010

40. Wirtz CR, Albert FK, Schwaderer M, Heuer C, Staubert A, Tronnier VM, et al: The benefit of neuronavigation for neurosurgery analyzed by its impact on glioblastoma surgery. Neurol Res 22:354-360, 2000

\section{Author Contributions}

Conception and design: Wolfsberger, Mert, Knosp, Widhalm.
Acquisition of data: Mert, Kiesel, Wöhrer, Martínez-Moreno, Minchev, Furtner, Widhalm. Analysis and interpretation of data: all authors. Drafting the article: Mert, Kiesel, Wöhrer, Widhalm. Critically revising the article: Wolfsberger, Mert, Kiesel, Wöhrer, Martínez-Moreno, Minchev, Knosp, Widhalm. Reviewed submitted version of manuscript: all authors. Approved the final version of the manuscript on behalf of all authors: Wolfsberger. Administrative/technical/material support: Wöhrer, Furtner, Widhalm.

\section{Correspondence}

Stefan Wolfsberger, Department of Neurosurgery, Medical University Vienna, Waehringer Guertel 18-20, A-1097 Vienna, Austria.email: stefan.wolfsberger@meduniwien.ac.at. 